\title{
30000 Usefulness of Stent-Assisted Coil Technical Embolization of Direct Traumatic Carotid-Cavernous Fistulas: Report of Three Patients and Review of Other Treatment Methods
}

Kenji Takahashi, ${ }^{1}$ Yui Nagata, ${ }^{1}$ Takurou Hashikawa, ${ }^{1}$ Hideki Sakai, ${ }^{1}$ Yoshihisa Matsumoto, ${ }^{1}$ Setsuko Nakagawa, ${ }^{1}$ and Yoshihisa Fukushima²

Objective: Direct traumatic carotid-cavernous fisulas (dtCCFs) exhibit a high blood flow velocity and are often difficult to be treated. We report three dtCCF cases in which disappearance of the dtCCF and preservation of the internal carotid artery (ICA) were achieved by stent-assisted coil embolization of the fistula. We report these cases and compare them with those previously reported using other treatments.

Case Presentations: In the first case, we performed coil embolization without stenting for the initial treatment. The cerebral venous reflux disappeared and the patient's symptoms were temporarily ameliorated. However, 5 months after treatment, an aneurysm-like finding around the fistula was noted on MRA. Additional coils and insertion of a neck-bridging stent were required to obliterate the dtCCF and the symptoms disappeared. In the second and third cases, we intended to use stents initially to achieve tight embolization of the fistulas, and obliteration was achieved.

Conclusion: Use of neck-bridging stenting for dtCCFs may be a reliable method to preserve the parent artery while achieving tight packing around the fistula.

Keywords > direct carotid-cavernous fistula, traumatic, stent-assisted, coil embolization

\section{Introduction}

Many direct carotid-cavernous fistulas (dCCFs) are caused by trauma (direct traumatic CCFs (dtCCF). This disorder is rare, accounting for approximately $0.17 \%-1.01 \%$ of patients with traumatic brain injury (TBI). ${ }^{1)}$ The purpose of treatment is to close the fistula. Currently, endovascular treatment is primarily selected, but most dtCCFs are high-flow-volume

\footnotetext{
${ }^{1}$ Department of Neurosurgery, St. Mary's Hospital, Kurume, Fukuoka, Japan

${ }^{2}$ Division of Cerebrovascular Medicine, St. Mary's Hospital, Kurume, Fukuoka, Japan
}

Received: July 29, 2019; Accepted: June 12, 2020

Corresponding author: Kenji Takahashi. Department of Neurosurgery, St. Mary's Hospital, 422, Tsubukuhonmachi, Kurume, Fukuoka 830-8543, Japan

Email: kjtk0823@outlook.jp

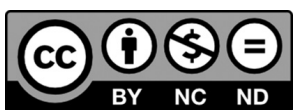

This work is licensed under a Creative Commons Attribution-NonCommercialNoDerivatives International License.

(C)2020 The Japanese Society for Neuroendovascular Therapy shunts and treatment is difficult in many cases. In countries where detachable balloons are not available, including Japan, other methods are adopted, but few studies have reported coil embolization with a neck-bridge stent for embolization of aneurysms. We report three patients in whom stent-assisted transarterial/-venous coil embolization led to complete cure with preservation of the parent blood vessel. We report the usefulness of this procedure and review reports of other treatment methods.

\section{Case Presentations}

Case 1: A 22-year-old woman.

Injury mechanism: Traffic accident. Dislocation/fracture of the 7th cervical vertebra and multiple costal fractures were noted, and laminoplasty and fixation were performed at the Department of Orthopedics.

Present illness/physical examination: Tinnitus developed 7 days after injury. Ocular hyperemia developed 2 weeks after injury. Diplopia developed 36 days after injury. She was referred/admitted to our hospital 40 days after injury. 

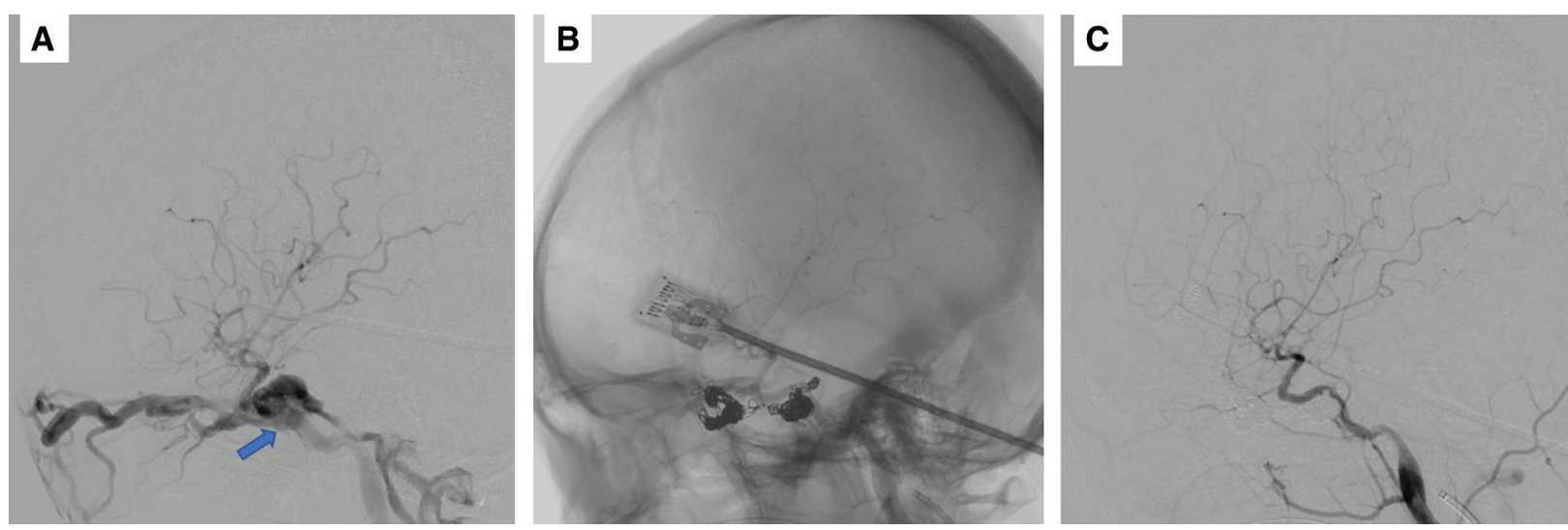

Fig. 1 Case 1. Initial treatment. (A) Lateral view on right internal carotid angiography. A fistula was present in the internal carotid artery-cavernous sinus region. Regurgitation to the superior ophthalmic and SMCVs was observed (arrow: fistula site). (B) After coil embolization. TVE was performed to reduce regurgitation to the superior ophthalmic and SMCVs using a coil. Subsequently, coil embolization (TAE and TVE) of the compartment around the cavernous sinus fistula was performed. (C) Final angiography on initial treatment. Regurgitation had disappeared and slight outflow to the pterygoid plexus was observed. SMCVs: superficial middle cerebral veins; TAE: transarterial embolization; TVE: transvenous embolization
Initial treatment: On the day of admission (Day 1), digital subtraction angiography (DSA) revealed a right dtCCF. The fistula was present in the cavernous portion of the internal carotid artery (ICA), measuring approximately $4 \mathrm{~mm}$. A balloon occlusion test (BOT) was conducted on Day 2, but occlusion of the proximal ICA led to restlessness 20-30 seconds after blockage, with a stump pressure of $0 \mathrm{mmHg}$. This suggested a fistula-mediated steal phenomenon. On Day 3, treatment was performed. The superficial middle cerebral vein (SMCV) and superior ophthalmic vein (SOV) had regurgitated into the cranial cavity. Initially, transvenous embolization (TVE) of these veins by coils was conducted via the inferior petrosal sinus (IPS). As a guiding system, a distal access catheter (DAC) was used. Adopting three steps of $8 \mathrm{~F}, 6 \mathrm{~F}$, and $4.2 \mathrm{~F}$, an $8 \mathrm{~F}$ FUBUKI $80 \mathrm{~cm}$ straight (Asahi Intecc, Aichi, Japan) was inserted into the right internal jugular vein (IJV), a 6F FUBUKI $100 \mathrm{~cm}$ angle (Asahi Intecc) into the proximal IPS, and a $4.2 \mathrm{~F}$ FUBUKI $120 \mathrm{~cm}$ (Asahi Intecc) into the distal IPS. A Headway $17150 \mathrm{~cm}$ microcatheter (Terumo Corporation, Tokyo, Japan) and CHIKAI $200 \mathrm{~cm}$ guidewire (Asahi Intecc) were used. Subsequently, TVE was performed through an approach to the periphery of the fistula, but the microcatheter deviated from this compartment during embolization, and transarterial embolization (TAE) was carried out by transarterially approaching the compartment via the fistula. As a guiding catheter, an OPTIMO $8 \mathrm{~F} 90 \mathrm{~cm}$ (Tokai Medical Products, Aichi, Japan) was used. The fistula was successfully closed (Fig. 1) and the patient was discharged.

Second treatment: Magnetic resonance imaging (MRI) 5 months after injury revealed an aneurysm-like finding in the compartment around the cavernous sinus fistula. Follow-up MRI after 5 weeks demonstrated slight enlargement and additional treatment was planned. This aneurysm-like finding suggested a pseudoaneurysm formed by coil compaction. We considered it difficult to transvenously approach this gap through the existing coil mass. Therefore, stentassisted coil embolization was performed and the fistula was successfully closed. A microcatheter was inserted using the jailed technique for the stent. An LVIS $4.5 \times 23 \mathrm{~mm}$ stent (Microvention, Aliso Viejo, CA, USA) was used for the following reasons: the fistula was present in the linear area of the ICA; and rectifying effects may be obtained. The total number of coils on the first and second sessions of embolization was 44 (26 at the cavernous sinus). The minimum diameter of the coils around the fistula was $3 \mathrm{~mm}$ (Fig. 2). There has been no recurrence during the 2-year-and-8month postoperative follow-up.

\section{Case 2: A 40-year-old man}

Injury mechanism: Facial blow. Present illness/physical examination: Exophthalmos, diplopia, and eye movement disorder were noted 33 days after injury. The patient was referred from the Department of Ophthalmology.

Treatment: A left dtCCF was observed. The fistula was present in the $\mathrm{C} 3$ portion of the ICA, measuring approximately $3 \mathrm{~mm}$. As described for Case 1, the SMCV and SOV had regurgitated into the cranial cavity. Initially, the SMCV was embolized and the SOV was partially embolized to reduce the shunt flow volume. For TVE, the same guiding system as used on initial treatment in Case 1 was inserted, and the same microcatheter and guidewire were used. Subsequently, TVE 

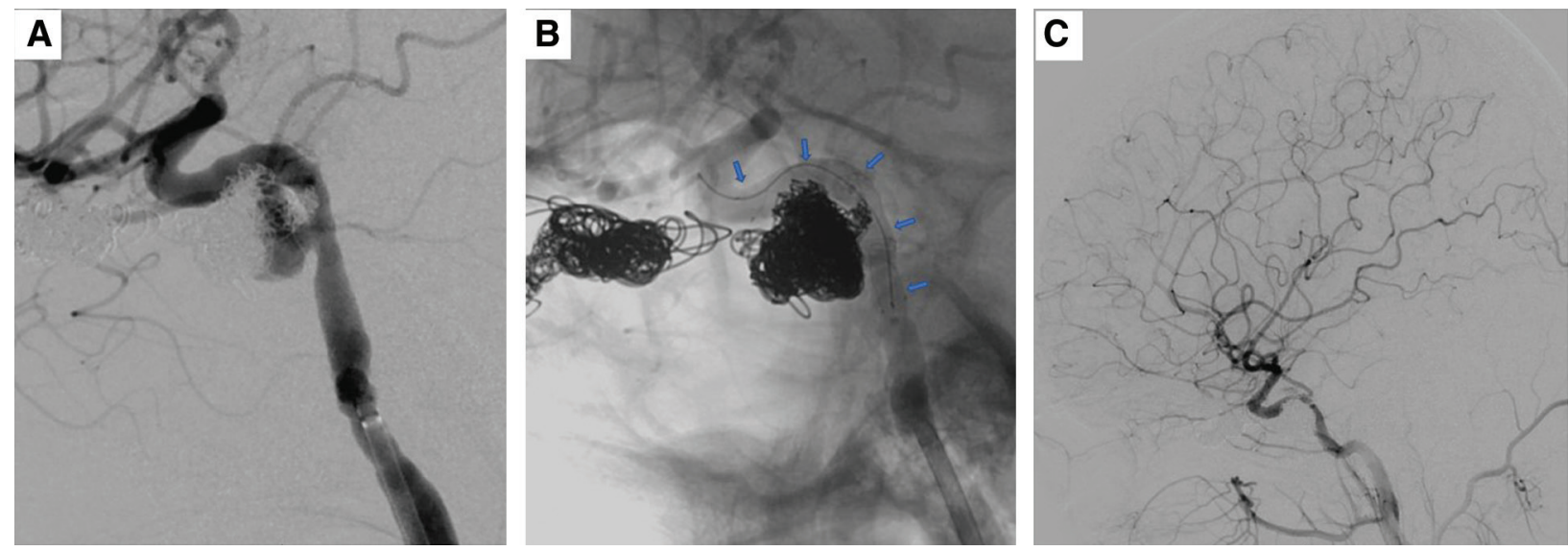

Fig. 2 Case 1. Second treatment. (A) Lateral view on right internal carotid angiography. There was no regurgitation into the cranial cavity, but compaction of a coil around the fistula was noted. A portion of the cavernous sinus resembled an aneurysm. Slight stenosis of the internal carotid artery, which may have been associated with the

of this compartment was attempted through an approach to the periphery of the cavernous sinus fistula. However, the compartment around the fistula had surrounded the ICA. We hypothesized the following: if this compartment is embolized with a coil, the ICA may be circumferentially surrounded; and even if neck plasty using a balloon is performed, there may be no method to confirm the presence of coil deviation to the ICA side after deflation. Therefore, stenting was selected to prevent this. An Excelsior XT-27 (Stryker, Kalamazoo, MI, USA) was guided into the distal ICA beyond the fistula through a $6 \mathrm{~F}$ Launcher $90 \mathrm{~cm}$ straight (Medtronic, Minneapolis, MN, USA) that had been placed in the ICA. Next, a steam-shaped Excelsior SL-10 (Stryker) was inserted into the cavernous sinus through the fistula. This was prepared as a strategy against unsuccessful TVE. As the stent, a Neuroform EZ $4.5 \times 30$ mm (Stryker) was used and a transarterial microcatheter was inserted using the jailed technique. Initially, the periphery of the fistula was transvenously embolized, but the catheter deviated to an extracompartmental area during embolization, and TAE was additionally performed, leading to the disappearance of regurgitation. The total number of coils was 31 (23 at the cavernous sinus). The minimum diameter of coils around the fistula was $2 \mathrm{~mm}$ (Fig. 3). There has been no recurrence during the 2-year-and-7-month postoperative follow-up.

Case 3: A 69-year-old woman.

Injury mechanism: Fall-related occipital bruise. Present illness/physical examination: On the day of injury, the patient was brought to our hospital by ambulance. Brain contusion and slight acute subdural hematoma were observed, and the compaction, was observed. (B) Lateral view on right internal carotid angiography. Magnified live image. After stent-assisted TAE with a coil (arrow: stent). (C) Lateral view on right common carotid angiography. Final image. Complete blockage of the fistula was achieved. TAE: transarterial embolization

patient was admitted. Consciousness was clear and conservative treatment was performed. However, right blepharoptosis was noted 2 days after admission. Subsequently, exophthalmos and oculomotor paralysis were observed. Furthermore, computed tomography (CT) demonstrated enlargement of edematous changes in the right frontal/temporal lobes, leading to a diagnosis of a right dtCCF.

Treatment: The fistula was present in the $\mathrm{C} 4$ portion of the ICA, measuring approximately $3 \mathrm{~mm}$. The SMCV, SOV, and superior petrosal sinus (SPS) to the petrosal vein had regurgitated into the cranial cavity. Initially, TVE was performed to stop regurgitation into the cranial cavity and the SOV was partially embolized to reduce the entire shunt flow volume, as described for Cases 1 and 2. For TVE, the same system adopted in Cases 1 and 2 was used. Next, TVE at the periphery of the fistula was attempted through an approach into the cavernous sinus, but it was impossible to transvenously reach this compartment. Therefore, TAE was selected, but the compartment around the fistula had surrounded the ICA, as described for Case 2. Thus, stent-assisted coil embolization was selected for similar reasons. We planned to insert a microcatheter into the cavernous sinus using the jailed technique for a stent, but as there was a possibility that a single catheter may deviate under insufficient embolization, the insertion of two microcatheters using the jailed technique was adopted. In addition to an OPTIMO 9F $90 \mathrm{~cm}$ (Tokai Medical Products) that had been placed in the ICA, a 5F Launcher $90 \mathrm{~cm}$ straight was inserted into the ICA. Through this microcatheter, an Excelsior XT-17 (Stryker) was guided into the ICA beyond the fistula for stenting. Subsequently, a Headway 17 and Excelsior SL-10 pre-shaped J (Stryker) 

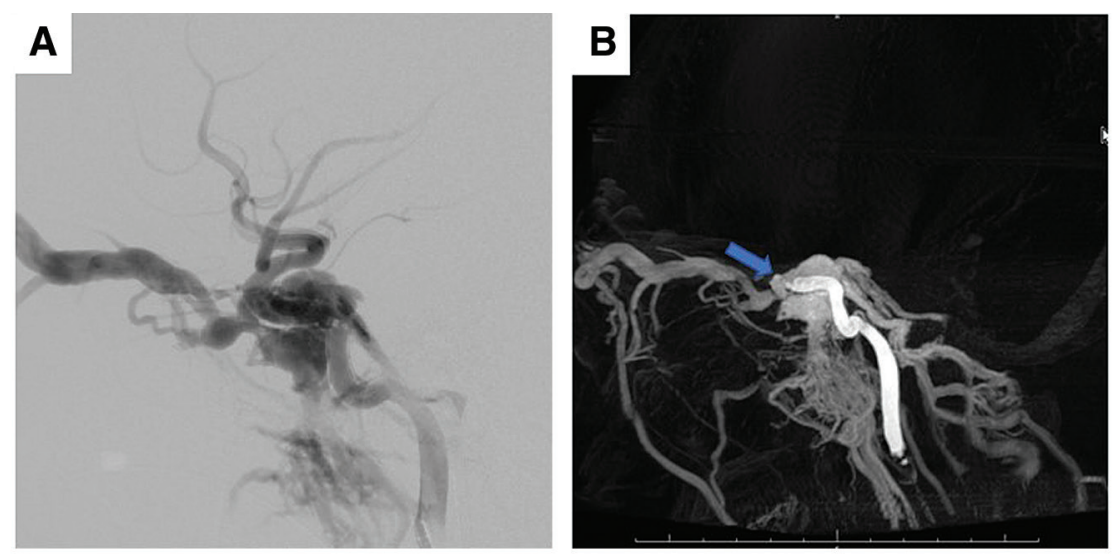

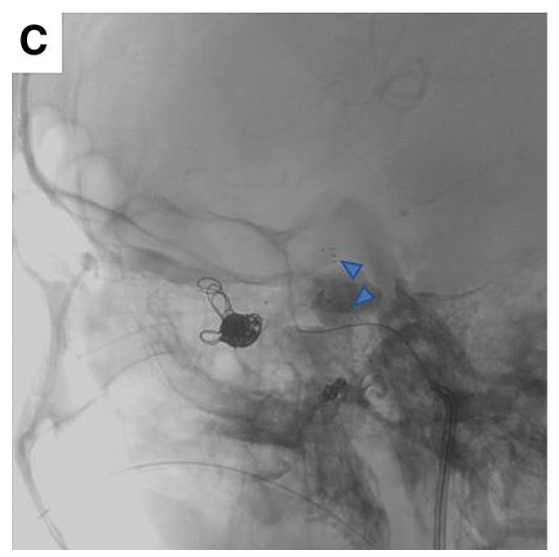

Fig. 3 Case 2. (A) Lateral view on left internal carotid angiography. A fistula was present in the internal carotid artery-cavernous sinus region. Regurgitation to the superior ophthalmic and SMCVs was observed. (B) MIP image of 3D-DSA. Imaging was conducted by dilating the balloon of a guiding catheter. The fistula site in the siphon area was clearly identified (arrow: fistula site). (C) Lateral cephalic view. TVE was performed to reduce regurgitation to the superior ophthalmic and SMCVs. Subsequently, a stent was inserted (arrow head: stent). (D) Lateral view on left internal carotid angiography. Live

were guided into the cavernous sinus for embolization. As the stent, a Neuroform Atlas $4.5 \times 21 \mathrm{~mm}$ (Stryker) was inserted. TAE was performed through the two microcatheters, leading to disappearance of the shunt. The total number of coils was 55 (18 at the cavernous sinus). The minimum diameter of coils around the fistula was $3 \mathrm{~mm}$ (Fig. 4). There has been no recurrence during the 1-year-and-3month postoperative follow-up.

\section{Discussion}

CCFs refer to a condition in which shunt blood flow through an abnormal fistula formed between the carotid artery or its branch and cavernous sinus due to some factor induces symptoms. Among CCFs, dCCFs are located in the ICA, directly flowing in the cavernous sinus. According to the Barrow classification, dCCFs are classified as type A. Most dCCFs are related to trauma (dtCCFs). Non-traumatic image. A microcatheter was transvenously guided and another microcatheter was transarterially (jail technique through a stent) guided. Through these microcatheters, TAE and TVE were performed to embolize the compartment around the cavernous sinus fistula. (E) Lateral view on left internal carotid angiography. Final image. The disappearance of regurgitation and complete blockage of the fistula were achieved. SMCVs: superficial middle cerebral veins; TAE: transarterial embolization; TVE: transvenous embolization; 3D-DSA: three-dimensional digital subtraction angiography

dCCFs account for approximately one-fourth of cases. ${ }^{1)}$ Most non-traumatic dCCFs are associated with cavernous sinus aneurysmal rupture, but other etiological factors include iatrogenic (endovascular treatment and transnasal pituitary surgery) and congenital (Ehlers-Danlos disease and fibromuscular dysplasia [FMD]) factors.

There are several methods of dCCF classification. In a classification based on the blood vessels responsible, CCFs are classified into direct (dCCF) and indirect CCFs. In a shunt-flow-volume-based classification, dCCFs are classified into high- and low-flow dCCFs. In an etiology-based classification, dCCFs are classified into idiopathic and non-idiopathic dCCFs. Furthermore, there is a fistula-size-based classification. When the fistula size is larger, the shunt flow volume is higher. Chi et al. reported that the fistula size was correlated with the interval from symptom development, and concluded that early detection/treatment aided in a complete cure and ICA preservation. ${ }^{2)}$ 

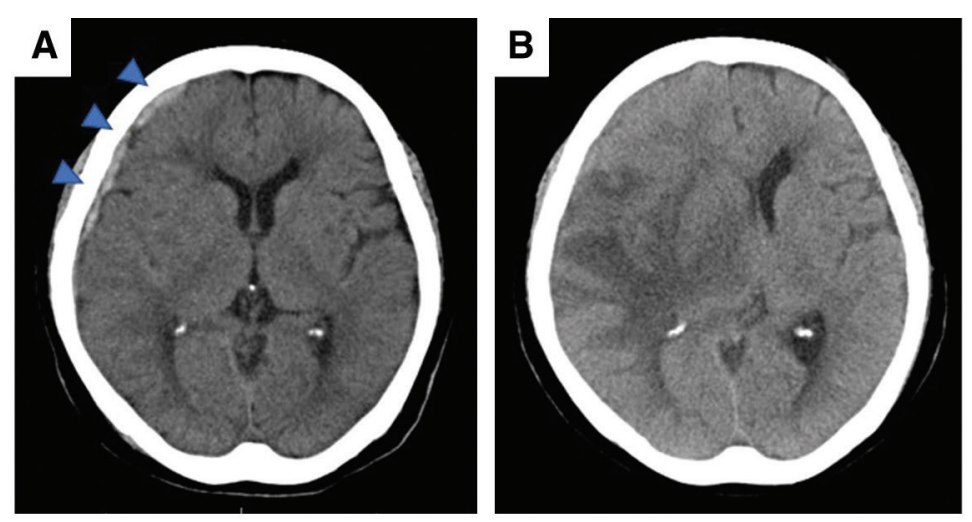
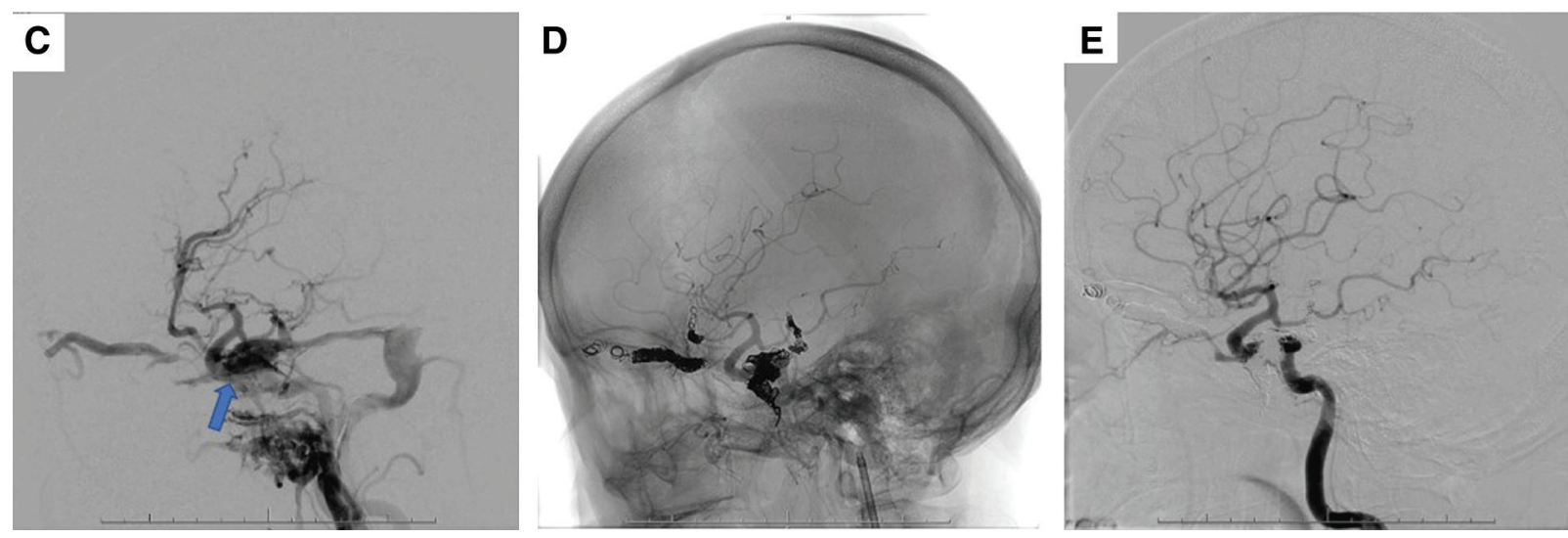

Fig. 4 Case 3. (A) Axial CT image. At the time of injury, slight subdural hematoma was observed below the right intracranial lamina (arrowhead: subdural hematoma). (B) Axial CT image. Marked edema of the right cerebral hemisphere and a midline shift were noted 13 days after injury. (C) Lateral view on right internal carotid angiography. A fistula was present in the internal carotid artery-cavernous sinus region. Outflow to the superior ophthalmic/SMCVs and SPS was observed (arrow: fistula site).

(D) Right internal carotid angiography. Live image. A stent was

In general, dtCCFs are high-flow shunts. Symptoms of dtCCFs are more marked than those of indirect CCFs, and their progression is more rapid. In addition to ocular symptoms, such as exophthalmos and eye movement disorder, increased intracranial pressure related to intracranial regurgitation may cause cerebral hemorrhage or ischemic symptoms related to a steal phenomenon, which is not observed in patients with indirect CCFs. In Case 3, only slight subdural hematoma and minor brain contusion were observed early after injury, but CT 13 days after injury revealed marked brain edema and a midline shift, suggesting cerebral hemorrhage (Fig. 4A and 4B). In Cases 1 and 2, BOT was performed before treatment, but when the ICA proximal to the fistula was occluded, the stump pressure was approximately $0 \mathrm{mmHg}$, and restlessness was noted 20-30 seconds after blockage. In the two patients, the development of a collateral pathway mediated by the anterior communicating artery was favorable, but contralateral blood observed in the cavernous sinus, and coils were observed in the outflow veins (petrosal vein, SPS, SMCV, and SOV) and compartment around the cavernous sinus fistula. (E) Lateral view on right internal carotid angiography. After stent-assisted coil embolization (TAE and TVE), complete blockage of the fistula was achieved. CT: computed tomography; SMCVs: superficial middle cerebral veins; SOV: superior ophthalmic vein; SPS: superior petrosal sinus; TAE: transarterial embolization; TVE: transvenous embolization

outflow to the venous side via the fistula was confirmed, reflecting relative ischemia related to the steal phenomenon. This suggested that treatment by simple ligation of the proximal ICA alone was impossible.

\section{dCCF: Changes in treatment methods}

In 1809, Traver et al. first reported ICA ligation for dCCFs. However, recurrence was frequent. Brooks (1930) and Lang et al. (1965) reported a method to open the cervical ICA and embolize a fistula by putting a muscle piece on the blood flow (Brooks method). The success rate was higher than that of ICA ligation, but issues, such as ICAocclusion-related cerebral infarction and incomplete occlusion, remained. ${ }^{3,4)}$ In 1973, Parkinson reported a method of directly repairing a fistula by direct surgery. However, there were limitations: technical difficulties, a large volume of blood loss, and the necessity of transient cardiac arrest. Furthermore, the incidence of neurological complications 
was high. ${ }^{5)}$ In 1971, Prolo and Hanbery proposed endovascular treatment for CCFs using a non-detachable balloon. ${ }^{6}$ In 1974, Serbinenko reported a method using a detachable balloon. ${ }^{7)}$ In 1985, supply was started in the United States and other countries under FDA guidance through improvements. Since then, this epoch-making method has been primarily selected, and many articles regarding its usefulness have been published.

\section{dCCF: Examination with respect to treatment methods}

The final goal of dCCF treatment is to reduce symptoms, but it is important to close the fistula with a low incidence of complications. In addition, the ICA should be preserved to prevent cerebral ischemia or aneurysm formation related to blood flow loading from a long-term point of view. The use of detachable balloons led to a high occlusion rate and a low incidence of complications. According to a review, patients in whom fistula occlusion is difficult account for approximately $10 \%$; therefore, detachable balloons are highly useful. ${ }^{1)}$ Furthermore, several studies reported that the double balloon technique improved the fistula passage and occlusion rates of balloons. ${ }^{8,9)}$ On the other hand, the ICA preservation rate was approximately $50 \%-90 \%$ in other studies. ${ }^{1,2,10-14)}$ In addition, after detachable balloons disappeared from the US market in 2004, they also became unavailable in Japan. Thereafter, coil embolization was primarily selected. However, Joshi et al. reported treatment using coils alone in 15 consecutive patients and reported an ICA preservation rate of $100 \%$, whereas the fistula closure rate was $80 \%$. According to their report, symptoms were ameliorated in 13 of 15 patients with chemosis, in 11 of 13 with proptosis, in 3 of 5 with papilledema, in 1 of 7 with ophthalmoplegia, in 9 of 11 with tinnitus, in 3 of 4 with visual loss, and in all 2 with neurological deficits ${ }^{15)}$; it may be difficult to achieve a treatment goal using coils alone. In this present situation, novel treatment methods have been attempted.

Several studies reported treatment using flow diverting stents (FDs). Ducruet et al. used FDs in 2 of 40 in whom treatment was possible among 42 patients with dCCFs, and reported the usefulness of combining coils with an FD.9) Furthermore, Nadaajah et al. ${ }^{16)}$ presented a patient in whom the use of two FDs led to fistula closure. Recently, Wendl et al. reported treatment with FDs. In 14 patients, 21 sessions were conducted, and FDs alone were used in five (covered stents or coils were combined with FDs in the others). The first session of treatment led to complete occlusion in three patients. Asymptomatic occlusion of the
ICA during the course was observed in 2 and symptoms were ameliorated in 10. The total number of FDs was 59.17) Based on these studies, for FD treatment without other devices, several FDs may be required to achieve fistula closure. In addition, neither symptom amelioration nor ICA preservation may be completely achieved. On the other hand, if an FD can be combined with coils, this treatment may be more effective through its rectifying effects.

Several studies have reported treatment using covered stents. He et al. noted successful insertion in 14 of 15 patients and that complete occlusion was achieved in 11, whereas incomplete occlusion was noted in 3. According to their report, occlusion was achieved during follow-up in the latter. There was no in-stent stenosis/occlusion during the course, and symptoms were ameliorated in 13 of the 15 patients. ${ }^{18)}$ Wang et al. used covered stents in $25 \mathrm{dtCCF}$ patients (27 lesions) in whom initial treatment did not lead to closure, and reported that the success rate of insertion was $100 \%$, although the total number of stents was 44 . According to their report, closure of $17 \mathrm{dtCCFs}$ was promptly achieved after stent insertion. Additional dilation was noted in six patients and an additional stent was required in four patients. ${ }^{19)}$ During follow-up, closure of all $27 \mathrm{dtCCFs}$ was achieved, but the ICA was only preserved in 23 patients. Furthermore, Tiewei et al. performed treatment using covered stents in eight dtCCF patients, and reported that fistula closure was promptly achieved after treatment in five and that the ICA preservation rate immediately after treatment was $100 \%$. They had no treatment-related complications or deaths. However, asymptomatic occlusion of the ICA was observed in one during follow-up and the total ICA preservation rate was $83.3 \%$. Furthermore, one patient died of acute myocardial infarction 10 days after treatment. ${ }^{20)}$ Covered stents may be useful for closing fistulas and preserving the ICA, as suggested for FDs. However, there are limitations: the ICA preservation rate is not $100 \%$, several stents may be required, the long-term oral administration of antiplatelet drugs is necessary, and stent insertion into a tortuous blood vessel may be difficult due to the hardness of the delivery system.

Several studies reported treatment using liquid embolic substances. Zenteno et al. presented five patients in whom dCCF treatment was performed using Onyx alone. TAE was selected in four and TVE was selected in one patient. They reported that closure was promptly achieved after treatment in four patients, whereas it was confirmed after 6 months in the remaining patient. However, regurgitation of ethylene vinyl alcohol (EVOH) copolymer to the ICA was 
observed in one patient treated by TAE, resulting in stenosis requiring additional stenting. Furthermore, cortical infarction developed in one patient. ${ }^{21)}$

Only a few studies have reported treatment using bare metal stents. Ducruet et al. performed treatment using bare metal stents in 6 of 42 patients with dCCFs, but there was no detailed description. ${ }^{9)}$ To our knowledge, no study has reported the results of treatment using a bare metal stent alone, although several studies adopted bare metal stents in some patients in a series of treatment using different devices.

However, the use of a neck bridge stent for aneurysm embolization may have several advantages. First, the ICA can be preserved and physicians responsible for endovascular treatment are familiar with this device. Furthermore, it can be readily delivered to the lesion site and the administration period of antithrombotic drugs may be shortened due to the bare metal stent. If a braided stent can be used, rectifying effects may be obtained, as demonstrated in Case 1 . In addition, a cavernous sinus peri-ICA compartment, which has become a larger space involving the artery to sinus due to a large fistula of the ICA, can be reduced to a smaller space by covering the fistula with a stent, thereby facilitating localized, dense coil packing using coils smaller than the fistula; fistula closure may be achieved and cranial nerve palsy may be prevented.

We initially stopped venous blood flow regurgitating into the cranial cavity, reduced SOV blood flow, and performed embolization at the periphery of the fistula. However, embolization of the compartment around the fistula should have been initially performed. As dtCCFs are highflow-volume shunts, the order of embolization is important. If a major vein with regurgitation is occluded before fistula occlusion, regurgitation to other veins may be increased, regurgitation to another latently existing minor vein may newly occur, or the cavernous sinus pressure may increase, deteriorating cranial nerve or ocular symptoms. Although there were no problems in the present cases, tight packing of an area adjacent to the fistula alone should be initially considered to avoid such risks. This procedure combined with stenting may be useful. Furthermore, the number of coils may be reduced, having medicoeconomical effects. On the other hand, high-flow-volume shunts may not be blocked by embolization at the fistula periphery alone even if a stent is used; it is necessary to secure an access route to a vein with regurgitation. Furthermore, it may be important to prepare a subsequent strategy, such as covered stent insertion, make a treatment plan in consideration of ICA trapping, and perform preoperative assessment (occlusion test at an area distal to the fistula site and examination for bypass, although these were not conducted in the present cases).

\section{Conclusion}

We report three patients in whom stent-assisted coil embolization was useful for the treatment of dtCCFs. The dtCCF occlusion rate after coil embolization is low and other treatment methods are not superior to treatment using a detachable balloon. Currently, this is not available and the use of a neck bridge stent should be considered for the following reasons: physicians responsible for endovascular treatment are familiar with this device, it can be readily delivered to the lesion site, the periphery of a fistula can be densely embolized while preserving the ICA, the administration period of antithrombotic drugs may be shortened, and this device may help prevent cranial nerve palsy or reduce the number of coils.

\section{Disclosure Statement}

The authors declare no conflicts of interest.

\section{References}

1) de Aguiar GB, Jory M, Silva JM, et al: Advances in the endovascular treatment of direct carotid-cavernous fistulas. Rev Assoc Med Bras (1992) 2016; 62: 78-84.

2) Chi CT, Nguyen D, Duc VT, et al: Direct traumatic carotid cavernous fistula: angiographic classification and treatment strategies. Study of 172 cases. Interv Neuroradiol 2014; 20 : 461-475.

3) Brooks B: The treatment of traumatic arteriovenous fistula. South Med J 1930; 23: 100-106.

4) Lang ER, Bucy PC: Treatment of carotid-cavernous fistula by muscle embolization alone: the brooks method. J Neurosurg 1965; 22: 387-392.

5) Parkinson D: Carotid cavernous fistula: direct repair with preservation of the carotid artery. Technical note. J Neurosurg 1973; 38: 99-106.

6) Prolo DJ, Hanbery JW: Intraluminal occlusion of a carotidcavernous sinus fistula with a balloon catheter. Technical note. J Neurosurg 1971; 35: 237-242.

7) Serbinenko FA. Balloon catheterization and occlusion of major cerebral vessels. J Neurosurg 1974; 41: 125-145.

8) Niu Y, Li L, Tang J, et al: Embolization of direct carotid cavernous fistulas with the novel double-balloon technique. Interv Neuroradiol 2016; 22: 201-205. 
9) Teng MM, Chang CY, Chiang JH, et al: Double-balloon technique for embolization of carotid cavernous fistulas. AJNR Am J Neuroradiol 2000; 21: 1753-1756.

10) Lewis AI, Tomsick TA, Tew JM: Management of 100 consecutive direct carotid-cavernous fistulas: results of treatment with detachable balloons. Neurosurgery 1995; 36: 239-244; discussion 244-245.

11) Ducruet AF, Albuquerque FC, Crowley RW, et al: The evolution of endovascular treatment of carotid cavernous fistulas: a single-center experience. World Neurosurg 2013; 80: 538-548.

12) Debrun G, Lacour P, Vinuela F, et al: Treatment of 54 traumatic carotid-cavernous fistulas. J Neurosurg 1981; 55: 678-692.

13) Goto K, Hieshima GB, Higashida RT, et al: Treatment of direct carotid cavernous sinus fistulae. Various therapeutic approaches and results in 148 cases. Acta Radiol Suppl 1986; 369: 576-579.

14) Higashida RT, Halbach VV, Tsai FY, et al: Interventional neurovascular treatment of traumatic carotid and vertebral artery lesions: results in 234 cases. AJR Am J Roentgenol 1989; 153: 577-582.

15) Joshi DK, Singh DD, Garg DD, et al: Assessment of clinical improvement in patients undergoing endovascular coiling in traumatic carotid cavernous fistulas. Clin Neurol Neurosurg 2016; 149: 46-54.

16) Nadaajah M, Power M, Barry B, et al. Treatment of a traumatic carotid-cavernous fistula by the sole use of a flow diverting stent. J Neurointerv Surg 2012; 4: e1.

17) Wendl CM, Henkes H, Martinez Moreno R, et al: Direct carotid cavernous sinus fistulae: vessel reconstruction using flow-diverting implants. Clin Neuroradiol 2017; 27: 493-501.

18) He XH, Li WT, Peng WJ, et al: Endovascular treatment of posttraumatic carotid-cavernous fistulas and pseudoaneurysms with covered stents. J Neuroimaging 2014; 24: 287-291.

19) Wang W, Li MH, Li YD, et al: Reconstruction of the internal carotid artery after treatment of complex traumatic direct carotid-cavernous fistulas with the willis covered stent: a retrospective study with long-term follow-up. Neurosurgery 2016; 79: 794-805.

20) Tiewei Q, Ali A, Shaolei G, et al: Carotid cavernous fistulas treated by endovascular covered stent grafts with follow-up results. Br J Neurosurg 2010; 24: 435-440.

21) Zenteno M, Santos-Franco J, Rodríguez-Parra V, et al: Management of direct carotid-cavernous sinus fistulas with the use of ethylene-vinyl alcohol (Onyx) only: preliminary results. J Neurosurg 2010; 112: 595-602. 